\title{
The Integration Model of Closed-Loop Supply Chain Resource Allocation Considering Remanufacturing
}

\begin{abstract}
Logistics resource planning is an integration model of materials requirement planning and distribution resource planning which is a resource allocation technology. It is a technology of satisfying both production material supply and resource allocation optimization which is based on inventory management. For the remanufacturing supply chain, recycling and rebuilding of products form a reverse materials movement loop which challenges the traditional logistics resource planning system. For the characteristics of reverse logistics of remanufacturing supply chain, we propose a closed-loop supply chain resource allocation model based on autonomous multientity. We focus on integration resource allocation model of materials requirement planning and distribution resource planning considering remanufacturing.
\end{abstract}

Keywords: remanufacturing, supply chain, reverse logistics, resource allocation, integration model

\section{Introduction}

Traditionally, supply chain is an opened-loop management process in the form of 'materials-production-productsgarbage.' Generally, to make sure the resources, like raw materials and funds, in the right position at the right time, the key mission of supply chain management is making some reasonable planning and scheduling. After the introduction of remanufacturing, the production organization has changed a lot. Moreover, as the development of enterprises, their business is more and more extensive. The more their business is extensive, the more business entities are included in supply chain. So the cost-control, resource allocation and reverse logistics are more and more complex. Therefore, constructing the integration model of closed-loop supply chain in the form of "materials-

Manuscript received January 1, 2016; accepted May 20, 2016

Xiao-qiu Shi (ه), Yan-yan Li, Wei Long

School of Manufacturing Science and Engineering, Sichuan University, Chengdu 610065, China.

Email: 315929207@qq.com production-products-recycling-remanufacturing-products' is inevitable. The planning and scheduling is a key link to reflect the efficiency and effectiveness of the supply chain (closed-loop supply chain). However, traditional methods, such as material requirement planning (MRP) method and distribution resource planning (DRP) method, are generally like a centralized management, which ignore the autonomy. Moreover, they are too much emphasis on the stability of the partnership, so they are difficult to adapt to rapidly changing of market environment (Han, Wu, \& Fan, 1998; Zhang, 1997). Fortunately, logistics resource planning (LRP) can solve the problem of production and circulation simultaneously. However, if an enterprise engages in production, circulation and remanufacturing at the same time, LRP is not enough again. Therefore, on the basis of LRP, this paper presents a hierarchical planning and scheduling model based on logistics and reverse logistics (LRP II) to meet the needs of new case in which the enterprises consider production, circulation and remanufacturing simultaneously.

\section{LRP and remanufacturing}

MRP is to achieve the effective allocation of material resources. So there is no excess work in process inventory (Bras \& Mcintosh, 1999; Gao, 2009; Wang, Liu, \& Huang, 2008; Zhu \& Pan, 2000). As the same idea of MRP in production community, using DRP in distribution community is appeared. However, there are not communication between MRO and DRP. Therefore, if an enterprise engages in both production and circulation, the traditional method (MRP/DRP method) is not enough to make it. Fortunately, there has been a logistics means of the material resource allocation called LRP for solving this problem (Li \& Long, 2012). The architecture of LRP is shown in Figure 1. So LRP is an integration model of MRP and DRP to solve the problem of considering production and circulation simultaneously.

For the sake of environment protection and resource allocation optimization, an enterprise must consider the remanufacturing. Remanufacturing is the rebuilding of a 


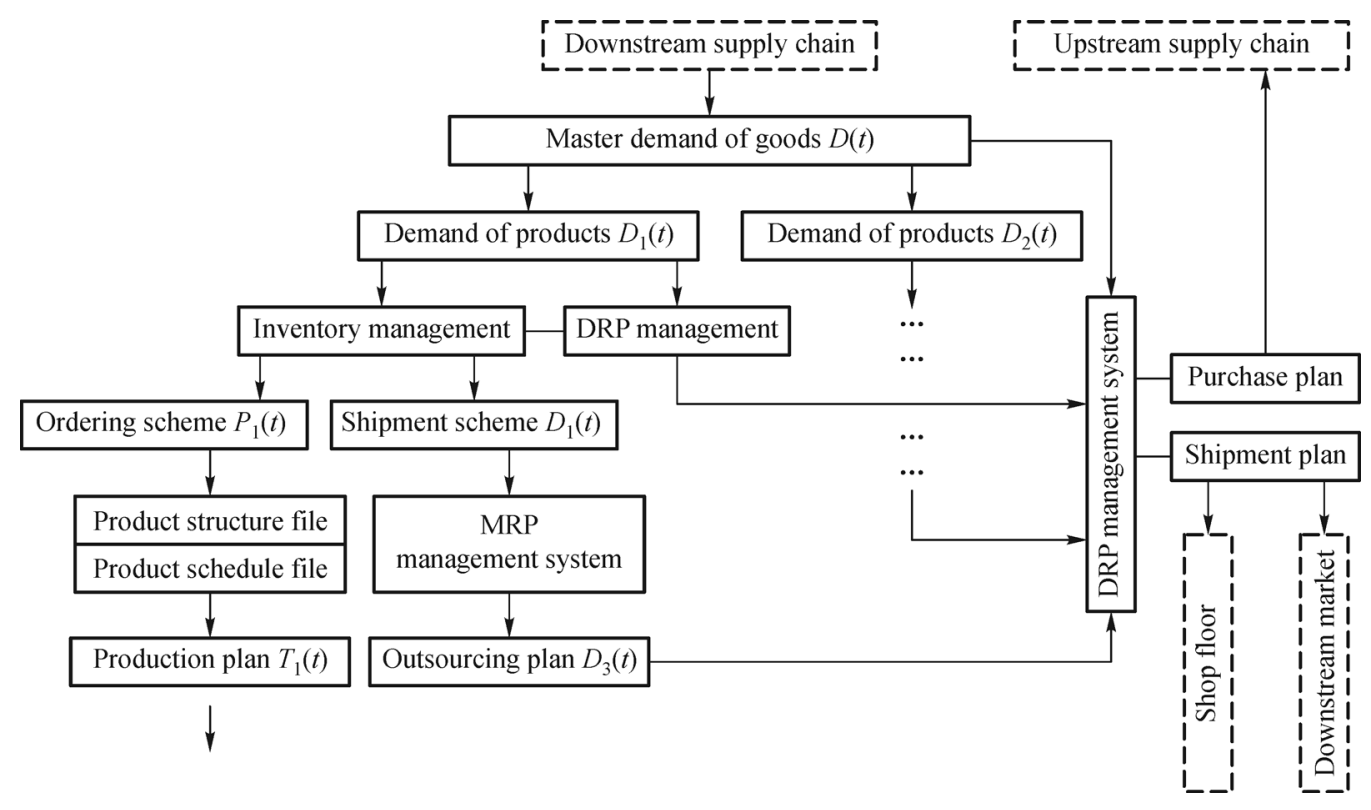

Figure 1. LRP architecture.

recycling product and it requires the repair or replacement of worn out parts. Remanufacturing is a form of a product recovery process that differs from other recovery processes in its completeness: a remanufactured machine should match the same customer expectation as new machines. Remanufacturing the returned products so that they perform as good as their new versions constitutes a higher form of recovery that is not used to the extent desired by the policy makers in many cases (Atasu, Van Wassenhove, \& Sarvary, 2009). Considering remanufacturing make us consider reverse logistics and then consider closed-loop supply chain. However, to solve the problem of production and circulation simultaneously, LRP considers the logistics means of DRP and MRP, and it does not consider remanufacturing and not considering reverse logistics. So this paper propose LRP II to solve this situation.

\section{The technological background of LRP II}

As the development of MRP, DRP and LRP, they not only have some advantages, but also expose their limitations.

The production system is relative regular and then the demand of materials is relative determinable, so MRP can make very precise material requirements planning. However, the implementation of this planning in the circulation is very complex, as there are a large number of random factors which affect the effectiveness of the MRP in turn. The same problem also happen to DRP, DRP can produce an accurate distribution requirements planning, but manufacturing plant and distribution center often appear randomness and do not guarantee the planning to be implemented smoothly, and then the distribution is restricted. The effectiveness of the DRP is affected (Chen, 1997; Guo, 2011; Yuan \& Liu, 2007). LRP is proposed to solve these problems.

With the economic development, enterprises need to survive the cruel completion, so the scope of business needs to be expanded. They not only produce the product but also be responsible for circulation. They not only produce single product but also produce variety of products, or they carry out a wide range of services. For the sake of environmental protection and cost-effectiveness, they also recycle products which are out of service rather than discard them directly. They must consider remanufacturing, that is, using the materials provided by recycled products together with the raw materials to produce products. So they must consider how to recycle products, that is, how to use reverse logistics. They also consider the closed-loop supply chains. Due to the fierce market competition, in order to further improve resource utilization, economic benefits and environmental protection, they not only consider the logistics means of LRP but also consider the reverse logistics means of LRP, that is, LRP II.

\section{The theory of LRP II}

Considering the remanufacturing, the basic idea of LRP II is that using the forward logistics and reverse logistics to overcome the boundaries of production and circulation and considering recycling products which are out of service. It contains several basic points in order.

(1) For the sake of environmental protection and green manufacturing, the LRP II not only reduces the cost but 
also reduce the discarded products. It is helpful to improve their social image. Moreover, for meeting the requirements of social market, it schedules the social resources. And for the production and circulation of products effectively, it also schedules the resources including the effective organization of resources from the production community and the internal enterprises.

(2) LRP II breaks the boundaries of production and circulation and it considers remanufacturing, so for the sake of cost-effectiveness, it allows the enterprises to plan and schedule the resources simultaneously in the inner and outside of the enterprises.

(3) Using various methods flexibly to break the region, sector, ownership boundaries and using a variety of business organizations, business methods, as well as all kinds of forward logistics and reverse logistics optimization method to achieve the efficient allocation of resources to improve economic efficiency.

(4) LRP II considers remanufacturing and then it includes reverse logistics and its randomness, such as, the time of recycled products is random, the physical position of recycled products is random, the amounts of recycled products is random and the quantities of recycled products is random. These qualities of randomness give the LRP II opportunities and challenges and then the implementation of LRP II is more complex. The more complex of LRP II, the probability of achieving more effective planning is bigger.

Flowing the above idea, the theory of LRP II is represented by architecture in Figure 2.

As can be seen from Figure 2, LRP II is actually the integration model of MRP and DRP considering remanufacturing and then considering both forward logistics and reverse logistics. The inputs of LRP II system are master file of social needs, product structure file, production capacity file, forward logistics capabilities document, reverse logistics capabilities document, production costs, supplier sourcing file and products form recycling system and so on. And then output the production plans, capacity requirements planning, delivery planning and ordering stock plans, transportation plans, forward logistics capacity requirements planning, reverse logistics capacity requirements planning and cost accounting.

The notations used here are shown in Table 1.

The implementation of LRP II can be divided into the following steps.

Step 1: Parameter is determined by the following equation.

$$
\begin{gathered}
Q(t)=Q(t-1)-D_{1}(t)-D_{4}(t) \\
P^{\prime}(t)=Q_{0}(t) \\
P_{1}(t)=P^{\prime}\left(t-t_{\mathrm{P}}\right)
\end{gathered}
$$

where $Q(t)$ is the current stocks, $Q(t-1)$ is the volume of the inventory, $Q_{0}(t)$ is the ordering bulk, $P^{\prime}(t)$ is the current plan, $t_{\mathrm{P}}$ is the lead time.

Step 2: Based $P_{1}(t)$ and $P_{2}(t)$ run MRP, we can get product put into scheme $T(t)$ and its components outsourcing plan $D_{3}(t)$.

Step 3: Put $D_{2}(t), D_{3}(t)$ and $D_{4}(t)$ to the DRP, resulting in $P(t)$ and $S(t)$, we can get $O_{\mathrm{S}}(t)$ and $O_{\mathrm{P}}(t)$.

$$
Q(t)=Q(t-1)-D_{2}(t)-D_{3}(t)+O_{\mathrm{S}}(t)+O_{\mathrm{P}}(t)
$$

When $Q(t)$ dropped below the reorder point, it is necessary to arrange an arrival, its offer is equal to an order $Q_{0}$.

$$
P^{\prime}(t)=Q_{0}
$$

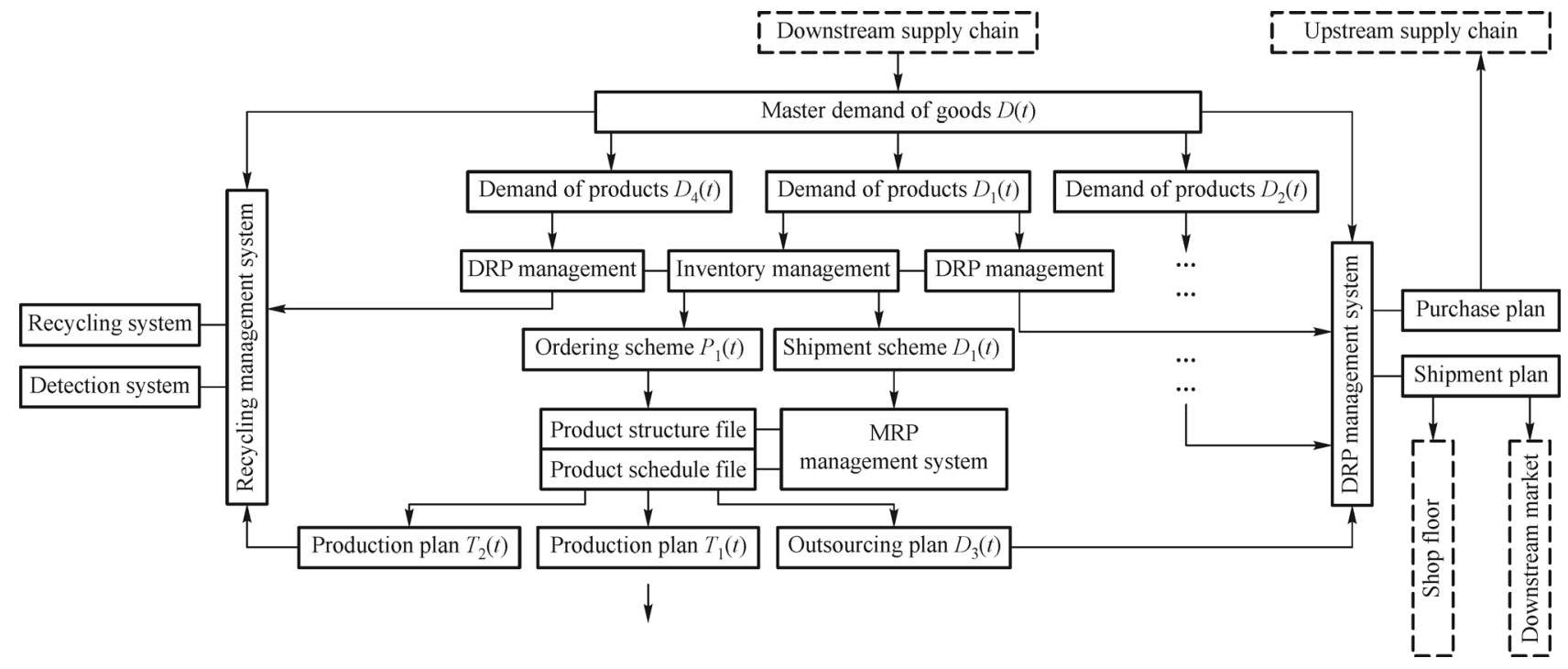

Figure 2. LRP II architecture. 
Table 1

Notations and its Explanation

\begin{tabular}{lc}
\hline Notations & Explanation \\
\hline$D(t)$ & Master demand schedule \\
$D_{1}(t)$ & A part of the main requirements document $D(t)$, which companies \\
can produce by themselves & Products are not produced by enterprise \\
$D_{2}(t)$ & Outsourcing plan \\
$D_{3}(t)$ & Products form recycling system which can be used by remanu- \\
$D_{4}(t)$ & facturing \\
$P(t)$ & Market order plan \\
$S(t)$ & Delivery scheme \\
$O_{\mathrm{s}}(t)$ & Product delivery on the way \\
$O_{\mathrm{P}}(t)$ & Product arrival or in-transit \\
$T_{1}(t)$ & Product plan whose materials are raw materials \\
$T_{2}(t)$ & Product plan whose materials are from recycling products \\
\hline
\end{tabular}

While ordering purchase plan:

$$
P(t)=P^{\prime}\left(t-t_{P}\right)
$$

Delivery plan $S(t)$ is:

$$
S\left(t-t_{\mathrm{S}}\right)=D(t)
$$

$t_{\mathrm{S}}$ is the delivery lead time.

Through these three-steps, we can use the LRP II to get more reasonable planning and scheduling of resources.

\section{Conclusions}

LRP is an integration model of MRP and DRP, which manages logistics and resource throughout the enterprises considering production and circulation. On the basis of LRP, this paper proposed LRP II which can get more reasonable planning and scheduling of social resources. It is proposed by improving the LRP by considering reverse logistics.

Reverse logistics has a large number of random factors. However, not every one of the random factors is considered in this paper. In the future research, the relationship of these random factors and how to handle them is necessary.

\section{References}

Atasu, A., Van Wassenhove, L.N., \& Sarvary, M. (2009). Efficient takeback legislation. Production and Operations Management, 18, 243258.

Bras, B., \& Mcintosh, M. W. (1999). Product, process and organization design for remanufacture - an overview research. Robots and Computer Integrated Manufacturing, 167-178.

Chen, Q. (1997). MRP II Manufacturing Resource Planning Basis. Beijing: Enterprise Management Publishing Group.

Gao, H. (2009). Workshop logistics intelligent control and warehouse management system research. Hubei: Huazhong University of Science and Technology.

Guo, J. (2011). Warehouse and MRP-II management $Q \& A$. Guangzhou: Guangdong Provincial Publishing Group.

Han, J., Wu, C., \& Fan, Y. (1998). Status and developing trends of supply chain modeling and management technology. CIMS, 4, 8-13.

Li, Y., \& Long, W. (2012). The integration model of supply chain resource allocation-LRP. International Asia Conference on Industrial Engineering and Management Innovation (IEMI2012) Proceedings, 1279-1285.

Wang, Q., Liu, F., \& Huang, H. (2008). Service oriented discrete workshop reconfigurable manufacturing execution system research. Computer Integrated Manufacturing, 4, 737-743.

Yuan, C., \& Liu, H. (2007). Logistics, Warehousing and Distribution Management. Beijing: Peking University Press.

Zhang, Y. (1997). MRP-II and its Application. Beijing: Tsinghua University Press.

Zhu, X., \& Pan, et al.. (2000). Mix the multi-objective optimization design variables of Pareto genetic algorithm to realize. Journal of Shanghai Jiao Tong University, 411-414. 